Article

\title{
Atomic Receiver by Utilizing Multiple Radio-Frequency Coupling at Rydberg States of Rubidium
}

\author{
Haiyang Zou ${ }^{1,2}$, Zhenfei Song ${ }^{2, *} \mathbb{0}$, Huihui Mu ${ }^{1,2}$, Zhigang Feng ${ }^{2}$, Jifeng $\mathrm{Qu}^{2}$ and \\ Qilong Wang ${ }^{1, *}$ \\ 1 Joint International Research Laboratory of Information Display and Visualization, Southeast University, \\ Nanjing 210000, China; zouhy@seu.edu.cn (H.Z.); skzxmhh@seu.edu.cn (H.M.) \\ 2 Center for Advanced Measurement Science, National Institute of Metrology, Beijing 100029, China; \\ fengzg@nim.ac.cn (Z.F.); qujf@nim.ac.cn (J.Q.) \\ * $\quad$ Correspondence: songzf@nim.ac.cn (Z.S.); northrockwql@seu.edu.cn (Q.W.)
}

Received: 24 January 2020; Accepted: 12 February 2020; Published: 16 February 2020

\begin{abstract}
Rydberg atoms have been extensively utilized in microwave measurement with high sensitivity, which has great potential in the field of communication. In this study, we discuss the digital communication based on a Rydberg atomic receiver under simultaneously coupling by resonant and near detuning microwaves. In addition, we verify the feasibility of the Rydberg atom-based frequency division multiplexing (FDM) in microwave communication. We demonstrate the principle and performance of the atom-based FDM receiver by applying amplitude modulation (AM) and frequency modulation (FM), respectively. To demonstrate the actual communication performance at different data transfer rates, we consider monochromatic images as an example. The experimental results show that the maximum acceptable data transfer rate of both AM and FM is about $200 \mathrm{kbps}$, whereas their maximum bit error rates (BER) is less than $5 \%$. When compared with the traditional electronic receiver, this atomic receiver, which is compatible with FDM, has numerous advantages, such as small size, low power consumption, and high sensitivity. Furthermore, this receiver has a strong ability of anti-electromagnetic interference, and the signals transmitted do not interfere with each other in different channels.
\end{abstract}

Keywords: Rydberg atoms; microwave communication; frequency-division multiplexing; antennas

\section{Introduction}

With the new developments in precision measurement technology, numerous atom-based sensors and communication devices have been proposed and verified in laboratories. Many new concepts and technologies of Radio Frequency Electric field (RF E-field) measurements in the quantum sensing domain have been presented, such as Rydberg atoms. Because of their high precision, sensitivity, and as the measurement results can be directly traced to the international system of units (SI) via atomic constant [1,2], they have great potential in many application scenarios. Rydberg atoms are some kinds of atoms with large principal quantum numbers [3]. Their large dc polarizabilities and microwave-transition dipole moments [4], Ref. [5] make them very suitable for the RF E-field measurement. Combining the electromagnetically induced transparency (EIT) [6,7] Autler-Townes (AT) [8] effects, measurement of the RF E-field with Rydberg atoms can be converted into frequency measurement. The sensitivity of the RF E-field has been observed to be less than $1 \mu \mathrm{V} \cdot \mathrm{cm}^{-1} \cdot \mathrm{Hz}^{-1 / 2}$ [9] under the predictable noise limit, and the measurement uncertainty has been observed to be less than $0.5 \%$ [2]. 
Rydberg atoms are also extensively employed in many fields, like high-resolution imaging, millimeter-wave/terahertz [10], and antenna measurement [11]. Owing to the high sensitivity of Rydberg atoms to RF E-field, the concept of wireless communication using Rydberg atoms has been recently proposed and verified. Deb et al. [12] proposed a new Rydberg atom-based wireless fiber-optic signal transmission system, which utilized the Rydberg atoms as a receiving antenna, and the signals were directly recovered at the baseband without any demodulation means. Meyer et al. [13] used Rydberg atoms as an optical antenna to realize the 8-state phase-shift-keying (PSK) digital communication protocol. Anderson et al. [14] demonstrated an atomic receiver for amplitude modulation (AM) and frequency modulation (FM) communication with a $3 \mathrm{~dB}$ bandwidth in the baseband of $100 \mathrm{kHz}$. Song et al. [15,16] investigated the feasibility of this atom-based receiver over a continuously tunable RF-carrier; the authors showed that the communication at a rate of $500 \mathrm{kbps}$ can be reliably performed within a tunable bandwidth of $200 \mathrm{MHz}$ near a $10.22 \mathrm{GHz}$ carrier. Holloway et al. [17] observed that the receiving system based on Rydberg atoms can detect and demodulate binary-phase-shift-keying (BPSK), quadrature-amplitude-modulation (QAM), and quadrature-phase-shift-keying (QPSK) signals. Due to numerous available resonant frequencies of the Rydberg states from $100 \mathrm{MHz}$ to sub-THz, it is possible to achieve the broadband communication by changing the coupling frequency between RF E-field and the Rydberg States. Therefore, Rydberg atoms are good candidates for microwave communication applications.

All the aforementioned communication experiments transmitted data over a single RF-carrier at atomic resonance frequency. As the development of wireless communications, spectrum resource is increasingly scarce, and interference between systems has become increasingly serious. It is of great necessity to find an optimal way to make better use of the limited spectrum resource. Using the abundant microwave-transition energy levels between Rydberg states, instead of recovering the time-varying signal on the specific RF-carrier frequency, we simultaneously couple resonant and detuning microwaves to verify the feasibility of dual-carrier communication and achieve frequency division multiplexing (FDM). This can improve spectral performance and allow real-time channel multiplexing.

In this paper, the feasibility of digital FDM communication is proposed, together with the atomic receiver by using multiple RF couplings. Additionally, we analyze the change of signal-to-noise ratio (SNR) by scanning the coupling laser frequency. The feasible experimental conditions which are selected by the SNR judgment are discussed. Finally, the image data reception is performed and practical indicators for AM and FM communication are discussed, respectively.

\section{Methods}

\subsection{Measurement Set-Up}

The details of how to use the Rydberg atoms as a receiving antenna for communication have been discussed in some previously conducted studies $[15,16]$. At both RF-resonance and RF-detuning, time-variant characteristics of complex signals could be captured by the change of the probe transmission, and modulation on a RF-carrier can cause synchronous modulation of the probe transmission. Thus, it is possible to retrieve the characteristics of the modulation signal by detecting the probe transmission in real-time.

FDM is a multiplexing technique that modulates multiple baseband signals onto different frequency carriers and then combines them to form a composite signal. In this paper, the communication of the atom-based receiver in the case of RF-resonance and RF-detuning carriers is proposed. Figure 1 illustrates the experimental set-up. A vapor cell containing rubidium $\left({ }^{87} \mathrm{Rb}\right)$ atoms was used as an active medium for real-time field sensing. ${ }^{87} \mathrm{Rb}$ atoms were excited to Rydberg states by counter-propagating probe laser and coupling laser via a two-photon transition. Meanwhile, through an external signal generator, two RF-carriers were amplitude or frequency modulated. Here, we use $\mathrm{CH} 1$ and $\mathrm{CH} 2$ to represent two RF-carriers, respectively. Then, they were coupled into the atomic vapor cell by the 
X-band horn antenna after the combiner, and different modulation parameters were selected for each modulation mode. The probe laser passing through the atomic vapor was detected by a photodiode, and recorded by a spectrum analyzer or the oscilloscope; signal components of the multiple basebands can be separated by a band-pass filter.

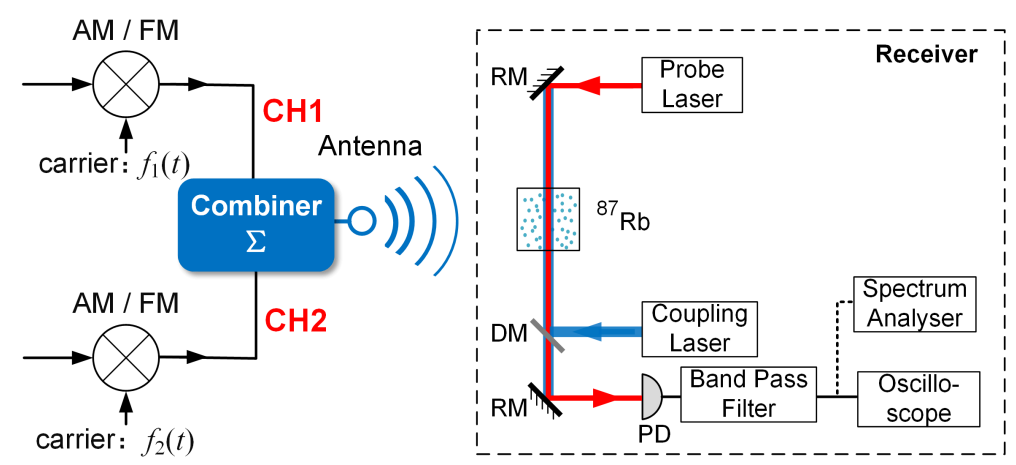

Figure 1. The experimental set-up. Frequency division multiplexing (FDM) receiver basing on Rydberg atoms is shown in the right part. Abbreviations: RM: reflector mirror; DM: dichroic mirror; PD: photodetector.

Figure 2a illustrates the experiment-related Rydberg energy levels. The probe laser $(\sim 780 \mathrm{~nm})$ generated from an external-cavity diode laser was frequency-locked to the atomic transition $5 \mathrm{~S}_{1 / 2}(\mathrm{~F}=2)$ and $5 \mathrm{P}_{3 / 2}\left(\mathrm{~F}^{\prime}=3\right)$, and the coupling laser $(\sim 480 \mathrm{~nm})$ generated from a high-power doubled-frequency diode laser system was frequency scanned across $5 \mathrm{P}_{3 / 2}\left(\mathrm{~F}^{\prime}=3\right)$ and Rydberg state $59 \mathrm{D}_{5 / 2}$. By regulating and controlling the laser, the phenomenon of a ladder three-level electromagnetically induced transparency (EIT) [18] appeared. When RF E-field at a resonant frequency of $10.22 \mathrm{GHz}$ between $59 \mathrm{D}_{5 / 2}$ and $60 \mathrm{P}_{3 / 2}$ was applied, it made EIT spectroscopy split, known as Autler-Townes (AT) effect. At the same time, another detuning RF E-field simulated by a microwave signal source was applied. When the RF E-field was frequency-detuned from the resonant transition, the observed AT-splitting spectroscopy became asymmetrical and two transmission peaks are not equal in height [16] (as shown in Figure 2b). In essence, the change of RF E-field frequency leads to the change of lineshape of AT-splitting, so both AM and FM baseband signals at multiple channels can be detected through the change of lineshape of a mixed AT-splitting [14].

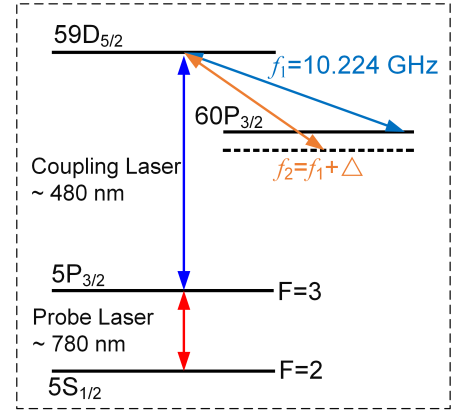

(a)

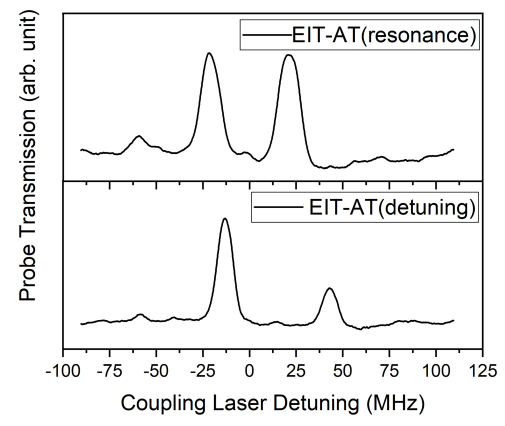

(b)

Figure 2. (a) Experiment-related energy levels. (b) RF-induced symmetric or asymmetric AutlerTownes splitting.

\subsection{Performance Test}

To verify the actual performance of the Rydberg atom-based FDM receiver, it was necessary to study the SNR, which is the main technical indicator for measuring the quality of communication systems. Moreover, to detect time-varying modulated signals, both the frequency of the probe and the coupling laser must be locked [15]. In this study, two modulated RF carriers for dual-channel 
communication were investigated. Signals received from a photodetector were separated by a high-pass filter and a low-pass filter. The modulation signals of the two channels were set as single-tone signals. The probe laser was frequency-locked to the $\mathrm{D} 2$ transition of ${ }^{87} \mathrm{Rb}$ atoms, and the best SNR was obtained by scanning the frequency of the coupling laser. SNR values of receiving signals of channel 1 $(\mathrm{CH} 1)$ and channel $2(\mathrm{CH} 2)$ were separately determined by a spectrum analyzer. The frequency of the modulation signal of $\mathrm{CH} 1$ was $15 \mathrm{kHz}$ with a peak-to-peak value of $3 \mathrm{~V}$, whereas the frequency of the modulation signal of $\mathrm{CH} 2$ was $10 \mathrm{kHz}$ with a peak-to-peak value of $4 \mathrm{~V}$. The purpose of adjusting the amplitude of the modulation signal was to make the amplitude of actually receiving signal identical. As the resonant responses of Rydberg atoms were dependent on the amplitude and frequency of RF E-field [19], to achieve optimal communication, the performance of the atomic receiver needs to be optimized to the best experimental SNR. The coupling laser was frequency-swept from $-80 \mathrm{MHz}$ to $80 \mathrm{MHz}$ respect to a resonance. The experimental results are presented in Figure 3. From Figure 3a, it can be seen that to achieve a high SNR in both channels for FM communication, the frequency of coupling laser should be locked away from the resonance (with zero coupling laser detuning). This is because, at this point, the dynamic range of probe transmission change caused by FM signals was the largest. For receiving AM signals, the frequency of the coupling laser should be locked at the resonance. The working points of coupling laser are indicated in Figure 3.
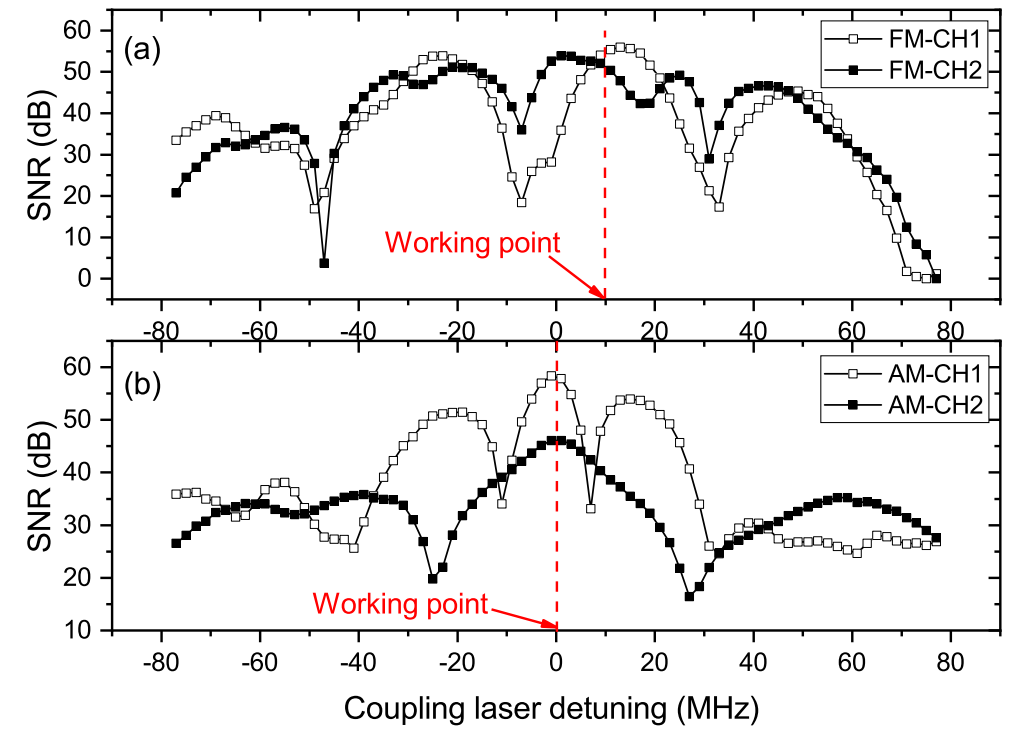

Figure 3. Observed signal-to-noise ratio (SNR) and working points of coupling laser. Results for dual-channel AM and FM communication are shown in (a) and (b), respectively.

In practical FDM communication, it is also very important that different channels should concurrently work together without any mutual interference. Interchannel interference (ICI) refers to the interference caused by signals in one or more channels in a given transmission channel. To analyze the ICI, we studied the variation of SNR of receiving signals with $\Delta_{R F}$, and the cross-talk between the actual signals obtained on the spectrum analyzer; here, $\Delta_{R F}$ represents the frequency difference between two carriers. A $10.22 \mathrm{GHz}$ carrier was applied to $\mathrm{CH} 1$, and a single-tone signal with an amplitude of $2.5 \mathrm{~V}$ and a frequency of $500 \mathrm{kHz}$ was modulated on the carrier. In addition, another carrier modulated by a single-tone signal with an amplitude of $2.5 \mathrm{~V}$ and a frequency of $550 \mathrm{kHz}$ was applied to $\mathrm{CH} 2$, and its frequency was scanned between 10.22 and $10.23 \mathrm{GHz}$ (i.e., $\Delta_{R F}$ was between 0 and $10 \mathrm{MHz}$ ). The corresponding results are shown in Figure 4. By observing the relative change in the SNR of receiving signals, the obtained SNR range of the two channels is from 26.4 to $28.2 \mathrm{~dB}$ when the $\Delta_{R F}$ is less than $10 \mathrm{MHz}$. By observing the signal from the spectrum analyzer, it can be seen that the two signals have a relatively pure spectrum even at a very close $\Delta_{R F}$ and there is no other spurious spectrum component, which can meet the requirements for practical 
communication and solve the problem of cross-talk. The same investigation was also carried out for AM communication and a similar conclusion has been reached. Traditional electronic-based receivers often produce spurious, harmonic, intermodulation, and other unnecessary signals, which will cause interference within the frequency band, whereas the atomic receiver will not cause cross-talk between signals while ensuring high SNR, so it has great potential in many applications.

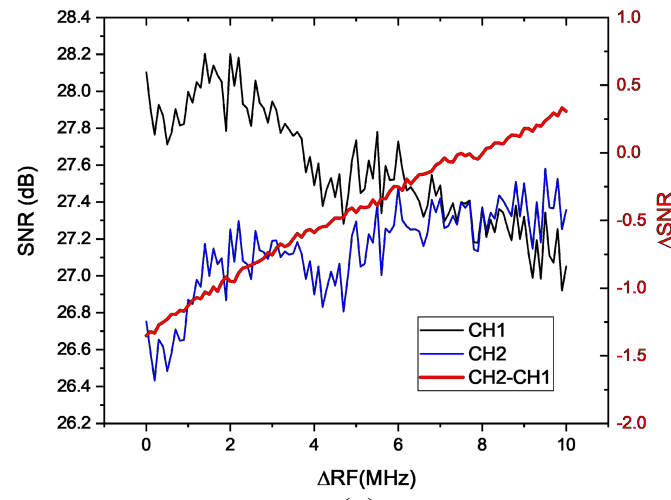

(a)

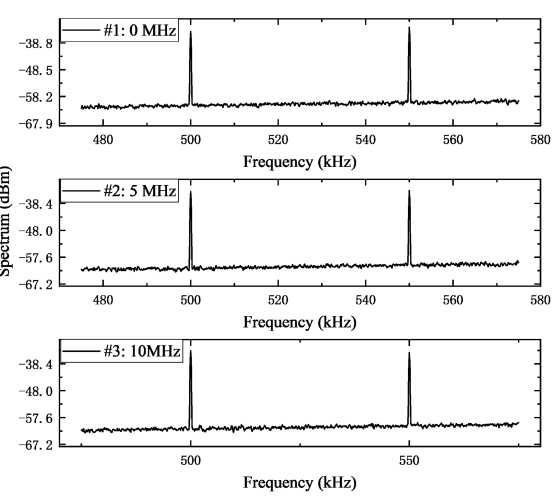

(b)

Figure 4. Interchannel interference performance of FM communication. (a) SNR of two channels changes with $\Delta_{R F}$ (b) Received signals when $\Delta_{R F}$ is 0,5 and $10 \mathrm{MHz}$ respectively.

\subsection{Wireless Reception of Image Data}

Based on different data transfer rates, more experiments were carried out to test the capability of data retrieving by the proposed atom-based FDM receiver. The transmitted images (letters " $\mathrm{A}$ ", "B", " $C$ ", and "D") are 36 by 36 pixel Portable Network Graphics (PNG) with lossless compression. Image data is encoded into a one-dimensional digital flow from corresponding two-dimensional array data, and then transmitted in different channels. Actually, images with different pixel sizes and formats can be used. The experimental probe laser power was $30 \mu \mathrm{W}$, whereas the coupling laser power was $100 \mathrm{~mW}$. The RF-carrier frequency of the CH1 was with a resonance frequency of $10.22 \mathrm{GHz}$ and a power of $3 \mathrm{dBm}$. The RF-carrier frequency of the $\mathrm{CH} 2$ had a detuning frequency of $10.25 \mathrm{GHz}$ and a power of $4 \mathrm{dBm}$. To achieve the port matching, the cable attenuation was calibrated before conducting the experiment. Moreover, power adjustment of the two channels made the actual antenna feeding power from the two channels identical, and both carriers were modulated by an external signal source. In the experiment of receiving FM signals, $\mathrm{CH} 1$ and $\mathrm{CH} 2$ transmitted the images of letters " $\mathrm{C}$ " and "D", respectively. The FM modulation depth in the experiment was $30 \mathrm{MHz}$. Figure 5 shows an image recovered by the proposed atomic receiver at various data transfer rates. It is clear that, with both modulation modes, the experimental results demonstrated an acceptable performance with a data transfer rate up to $500 \mathrm{kbps}$.

In digital transmission, bit error ratio (BER) was used to study the probability of receiving bits of data stream on the communication channel, which is often due to noise, interference, distortion, or bit synchronization error. In our experiment, a classical definition of BER is used, that is, the number of bit errors divided by the total transferred bits. BER values of different channels and modulation are shown in Figure 6. Generally, BER for data transfer in the detuning carrier was higher than that in the resonance situation. In the experiment of receiving the AM signal, the BER of the two channels can be kept at nearly 0 when the data transfer rate was less than $100 \mathrm{kbps}$. For receiving FM signals, the BER was close to 0 when the data transfer rate was less than $300 \mathrm{kbps}$, and even with the data transfer rate going up to $500 \mathrm{kbps}$, the BER increased to approximately $2 \%$. 


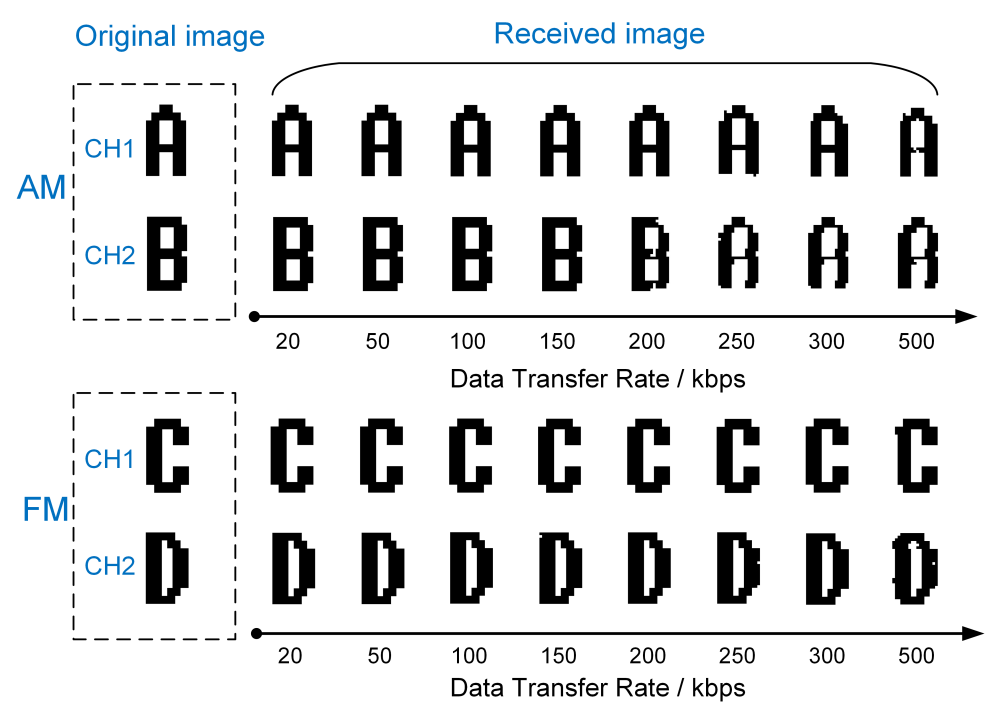

Figure 5. Test results of the proposed atomic FDM receiver for image data reception at different data transfer rates.

In the latest released technical specification document (3GPP TS 23.107 V15.0.0) within the 3rd-Generation Partnership Project (3GPP), the BER value ranges for Universal Mobile Communication System (UTMS) bearer service attributes are recommended. For some applications of Class 2 bits, a higher BER (5\%) might be feasible. The experimental results show that the maximum acceptable data transfer rate of both AM and FM is $200 \mathrm{kbps}$, whereas their maximum BER is less than $5 \%$.

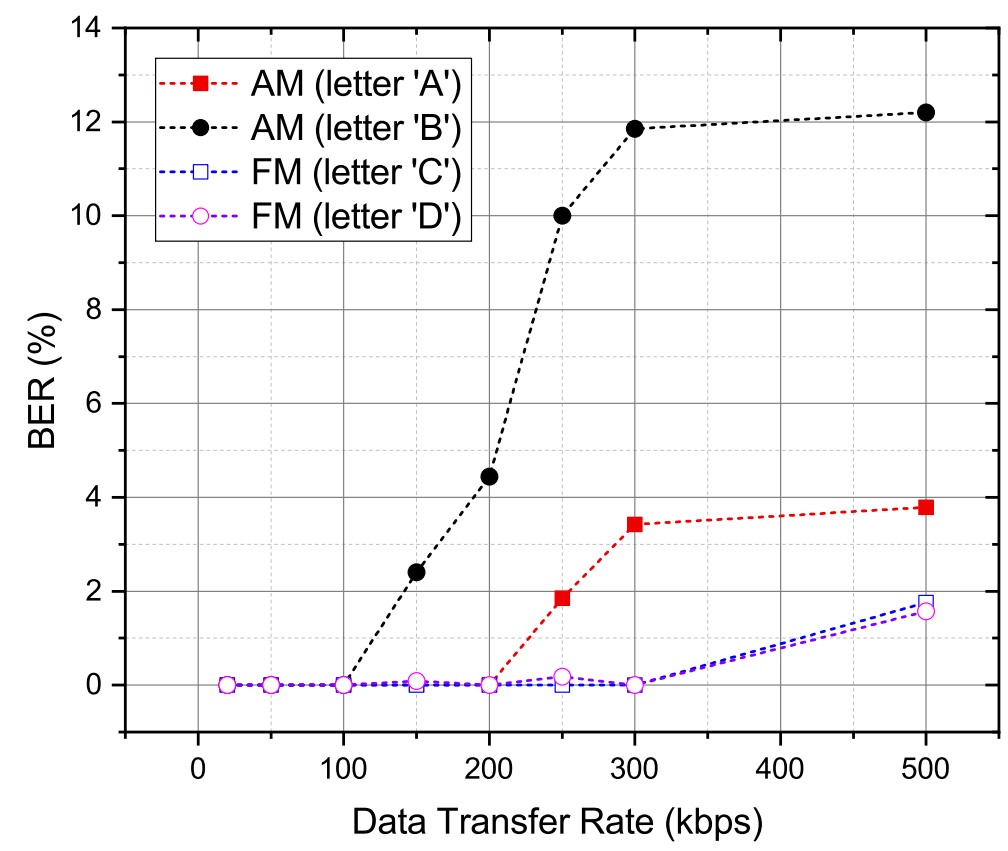

Figure 6. Bit error ratio (BER) for image data reception at different channels.

\section{Conclusions}

In this paper, the feasibility of an atom-based FDM receiver was discussed in detail through the method of dual RF-carrier. Various factors affecting the communication quality, such as BER and SNR, were studied. Our new scheme is of great significance to the field of atomic communication. By using Rydberg atom as a receiving antenna, the AM and FM signal was directly captured by the fast photodetector and can be obtained without any demodulation means. A demo experiment of image 
data reception at two channels has shown that the maximum acceptable data transfer rate was $200 \mathrm{kHz}$ with a BER of less than 5\%, for both AM and FM communication. Comparing with the traditional electronic-based receivers, the new atomic approach can realize the communication with high sensitivity and weak signal detection ability. Moreover, the signals transmitted by the two channels worked in parallel, and do not interfere with each other under a certain bandwidth, which greatly saved the spectrum resources. Based on the above excellent performance, these atomic receivers have great development potential in numerous applications. In the future, the performance of the atomic receiver will also be optimized for various communication technologies in our subsequent experiments.

Author Contributions: Conceptualization, Z.S.; Formal analysis, H.Z., H.M., Z.F., J.Q., Q.W., and Z.S.; Methodology, Z.S., H.Z., and Z.F.; Writing—original draft, H.Z.; Writing—review \& editing, Z.F., Q.W., and Z.S. All authors have read and agreed to the published version of the manuscript.

Funding: This work was funded by the National Key Research and Development Program of China (Grant No. 2016YFF0200104).

Conflicts of Interest: The authors declare no conflicts of interest.

\section{References}

1. Sedlacek, J.A.; Schwettmann, A.; Kübler, H.; Löw, R.; Pfau, T.; Shaffer, J.P. Microwave electrometry with Rydberg atoms in a vapour cell using bright atomic resonances. Nat. Phys. 2012, 8, 819-824. [CrossRef]

2. Holloway, C.L.; Simons, M.T.; Gordon, J.A.; Dienstfrey, A.; Anderson, D.A.; Raithel, G. Electric field metrology for SI traceability: Systematic measurement uncertainties in electromagnetically induced transparency in atomic vapor. J. Appl. Phys. 2017, 121, 233106. [CrossRef]

3. Saffman, M.; Walker, T.G.; Mølmer, K. Quantum information with Rydberg atoms. Rev. Mod. Phys. 2010, 82, 2313. [CrossRef]

4. Miller, S.A.; Anderson, D.A.; Raithel, G. Radio-frequency-modulated Rydberg states in a vapor cell. New J. Phys. 2016, 18, 053017. [CrossRef]

5. Wade, C.G.; Šibalić, N.; de Melo, N.R.; Kondo, J.M.; Adams, C.S.; Weatherill, K.J. Real-time near-field terahertz imaging with atomic optical fluorescence. Nat. Photonics 2017, 11, 40. [CrossRef]

6. Weatherill, K.; Pritchard, J.; Abel, R.; Bason, M.; Mohapatra, A.; Adams, C. Electromagnetically induced transparency of an interacting cold Rydberg ensemble. J. Phys. At. Mol. Opt. Phys. 2008, 41, 201002. [CrossRef]

7. Bason, M.; Mohapatra, A.; Weatherill, K.; Adams, C. Electro-optic control of atom-light interactions using Rydberg dark-state polaritons. Phys. Rev. A 2008, 77, 032305. [CrossRef]

8. Abi-Salloum, T.Y. Electromagnetically induced transparency and Autler-Townes splitting: Two similar but distinct phenomena in two categories of three-level atomic systems. Phys. Rev. A 2010, 81, 053836. [CrossRef]

9. Fan, H.; Kumar, S.; Sedlacek, J.; Kübler, H.; Karimkashi, S.; Shaffer, J.P. Atom based RF electric field sensing. J. Phys. B At. Mol. Opt. Phys. 2015, 48, 202001. [CrossRef]

10. Gordon, J.A.; Holloway, C.L.; Schwarzkopf, A.; Anderson, D.A.; Miller, S.; Thaicharoen, N.; Raithel, G. Millimeter wave detection via Autler-Townes splitting in rubidium Rydberg atoms. Appl. Phys. Lett. 2014, 105, 024104. [CrossRef]

11. Song, Z.; Feng, Z.; Liu, X.; Li, D.; Zhang, H.; Liu, J.; Zhang, L. Quantum-based determination of antenna finite range gain by using Rydberg atoms. IEEE Antennas Wirel. Propag. Lett. 2017, 16, 1589-1592. [CrossRef]

12. Deb, A.; Kjærgaard, N. Radio-over-fiber using an optical antenna based on Rydberg states of atoms. Appl. Phys. Lett. 2018, 112, 211106. [CrossRef]

13. Meyer, D.H.; Cox, K.C.; Fatemi, F.K.; Kunz, P.D. Digital communication with Rydberg atoms and amplitude-modulated microwave fields. Appl. Phys. Lett. 2018, 112, 211108. [CrossRef]

14. Anderson, D.A.; Sapiro, R.E.; Raithel, G. An atomic receiver for AM and FM radio communication. arXiv 2018, arXiv:1808.08589.

15. Song, Z.; Zhang, W.; Liu, X.; Zou, H.; Zhang, J.; Jiang, Z.; Qu, J. Quantum-Based Amplitude Modulation Radio Receiver Using Rydberg Atoms. In Proceedings of the 2018 IEEE Globecom Workshops (GC Wkshps), Abu Dhabi, UAE, 9-13 December 2018; pp. 1-6. 
16. Song, Z.; Liu, H.; Liu, X.; Zhang, W.; Zou, H.; Zhang, J.; Qu, J. Rydberg-atom-based digital communication using a continuously tunable radio-frequency carrier. Opt. Express 2019, 27, 8848-8857. [CrossRef] [PubMed]

17. Holloway, C.L.; Simons, M.T.; Gordon, J.A.; Novotny, D. Detecting and Receiving Phase Modulated Signals with a Rydberg Atom-Based Mixer. IEEE Antennas Wirel. Propag. Lett. 2019, 18, 1853-1857. [CrossRef]

18. Boller, K.J.; Imamoğlu, A.; Harris, S.E. Observation of electromagnetically induced transparency. Phys. Rev. Lett. 1991, 66, 2593. [CrossRef] [PubMed]

19. Berman, P.R.; Malinovsky, V.S. Principles of Laser Spectroscopy and Quantum Optics; Princeton University Press: Princeton, NJ, USA, 2011.

(C) 2020 by the authors. Licensee MDPI, Basel, Switzerland. This article is an open access article distributed under the terms and conditions of the Creative Commons Attribution (CC BY) license (http://creativecommons.org/licenses/by/4.0/). 